\title{
Hybridization of Monarch Butterfly and Grey Wolf Optimization for Optimal Routing in VANET
}

\author{
G. Tony Santhosh, S. Dhandapani
}

\begin{abstract}
The routing process in Vehicular Ad hoc Networks (VANET) remains a more demanding task in city backgrounds. Identifying an optimal end-to-end path that satisfies reduced overhead and delay control is still facing a lot of difficulties and limitations in recent days. These limitations are owing to the increased movement of vehicles, the repeated failures of a path, and the varied obstacles that might have an effect on the consistency of the data routing and transmission. Hence, this paper intends to present an enhanced VANET routing model by considering the network quality metrics including congestion, travel, collision and QoS awareness cost. Accordingly, in the proposed work, a cost model is modeled as the solution for the vehicle routing problem by taking into account the above-mentioned constraints. For determining the optimal route, this research work establishes a new hybrid algorithm known as Grey Updated Butterfly Operator (GU-BO) that links both the concepts of Monarch Butterfly Optimization (MBO) Algorithm and Grey Wolf Optimization (GWO). Finally, the performance of the implemented approach is compared over other conventional approaches with respect to congestion and cost analysis, and proves its superiority of proposed work over others.
\end{abstract}

Keywords - Collision cost; Cost analysis; MBO optimization; Optimal routing; VANET.

Nomenclature

\begin{tabular}{|l|l|}
\hline Abbreviation & \multicolumn{1}{|c|}{ Description } \\
\hline VANET & Vehicular Ad hoc Networks \\
\hline MANET & Mobile Ad hoc Network \\
\hline V2V & vehicle to vehicle \\
\hline LBS & Location-Based Services \\
\hline GPS & Global Positing System \\
\hline MAC & Medium Access Control \\
\hline AODV & Ad hoc on-Demand Distance Vector \\
\hline DSR & Dynamic Source Routing \\
\hline TROPHY & $\begin{array}{l}\text { Trustworthy VANET ROuting with grouP } \\
\text { autHentication keYs }\end{array}$ \\
\hline AMGRP & $\begin{array}{l}\text { AHP-dependent Multimetric Geographical Routing } \\
\text { Protocol }\end{array}$ \\
\hline AHP & Analytical Hierarchical Process \\
\hline PDR & packet delivery rate \\
\hline SES & Sampling-based Estimation Scheme \\
\hline WSN & Wireless Sensor Network \\
\hline ACO & Ant Colony Optimization \\
\hline HMR & Hadoop Map Reduce \\
\hline GF & Greedy Forwarding \\
\hline DCF & Distributed Coordination Function \\
\hline FIS & Fuzzy Inference System \\
\hline
\end{tabular}

Revised Manuscript Received on December 30, 2019.

* Correspondence Author

G. Tony Santhosh, Assistant Professor Department of ECE, Alpha College of Engineering, Chennai, India

Dr. S. Dhandapani, Professor Department of ECE, Saveetha Engineering, College, Chennai, India

(C) The Authors. Published by Blue Eyes Intelligence Engineering and Sciences Publication (BEIESP). This is an open access article under the CC BY-NC-ND license (http://creativecommons.org/licenses/by-nc$\underline{\mathrm{nd} / 4.0 /)}$

\begin{tabular}{|l|l|}
\hline RSS & Received Signal Strength \\
\hline QoS & Quality of Service \\
\hline MBO & Monarch Butterfly Optimization \\
\hline GWO & Grey Wolf Optimization \\
\hline MC-JA & Mean Computing Jaya Algorithm \\
\hline PSO & Particle Swarm Optimization \\
\hline FF & Firefly algorithm \\
\hline WAO & Whale Optimization Algorithm \\
\hline GU-BO & Grey Updated Butterfly Operator \\
\hline e2e & end-to-end \\
\hline
\end{tabular}

\section{INTRODUCTION}

VANET is a particular category of MANET. In VANET each vehicle performing as node acts as a router to switch over data among various nodes in the network [1]. VANETs are modeled to offer vehicle communication[3]. Two kinds of communication are feasible in VANET i.e. roadside to a vehicle and $\mathrm{V} 2 \mathrm{~V}$ communication. In VANET, there are stable network nodes that are deployed in the structure of roadside units [6]. These kinds of networks are modeled to deal with road traffic, safety purposes, driver control and LBS. In VANETs, power utilization and storage capacity are not restricted and the node's position could be portrayed by means of GPS[7]. A self-organized system could be formed by the VANETs, but it is not required for a premeditated infrastructure[9]. There is a restricted coverage level for every wireless network of the vehicle, which exists from 100-300 meters and therefore e2e communication is feasible across a huge distance.

To pass on a message to a destination node in VANET, it needs messages to deliver through numerous nodes[11] [13]. VANETs could offer cost-effective and scalable solutions for appliances namely, context-aware advertisement, traffic safety, and dynamic route planning by means of wireless communication at short ranges [14] [16]. For functioning appropriately, these appliances necessitate proficient routing protocols. VANETs networks further include a better perspective of extensive usage since it is low-cost, scalable, and offers increased bandwidth when distinguished with cellular communication [17]. On the other hand, it also comprises certain disadvantages associated with the comparatively increased vehicle speed that causes frequent and fast variations in topology. Proficient support of routing practices in vehicular surroundings is further meeting up with numerous problems such as MAC, accessible bandwidth evaluation, increased mobility, exposed and hidden nodes crisis, and speedy handover, movement of a node, rapid speed, blockages, and heterogeneous vehicles support [18]-[20].

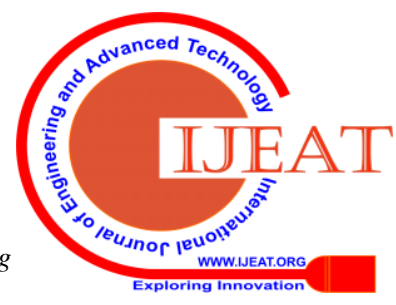




\section{Hybridization of Monarch Butterfly and Grey Wolf Optimization for Optimal Routing in VANET}

The major difficulty with the routing protocols such as AODV routing,

DSR, etc in VANETs surroundings is their instability in routing[21] [22]. The conventional node-centric observation of routes (that is, a recognized route between the destination and source) leads to numerous malfunctioning routes in the existence of high mobility of VANETs [23]. The major contribution of the proposed routing model is as follows:

The major contribution of this paper is depicted below.

1. This paper intends to present an enhanced VANET routing with the aid of a new GU-BO model by concerning the routing parameters namely, congestion cost, travel cost, collision cost, and QoS awareness cost.

2. Here, in the presented scheme, a cost model is modeled for handling the vehicle routing problems by considering the abovementioned cost parameters along with the Fuzzification of QoS.

3. The main aim of the proposed model is to discover the optimal route that minimizes the routing cost model, for which a hybrid algorithm known as GU-BO is introduced.

4. Finally, the performance of the adopted GU-BO scheme is compared over other conventional approaches and the superior outcomes are achieved.

The overall organization of the work is as follows: Section II portrays the literature work. Section III describes the objective model for optimal routing in VANET. Section IV presents the optimal route selection in VANET using the GU-BO algorithm. Section $\mathrm{V}$ portrays the experimental outcomes, and Section VI concludes the paper.

\section{LITERATURE REVIEW}

\section{A. Related works}

In 2018, Cirne et al. have introduced a set of protocols, TROPHY, to deal with routing information authentication in VANET. Legal nodes obtain TROPHY messages recursively, which permit them to revive their cryptographic substance and the authentication keys were updated in the network. In addition, a system was included to retrieve the data from any illegal physical contact and leak of the entire such information promptly, without necessitating the need for human involvement on restructuring the devices[1].

In 2017, Sami et al. have introduced an expansion on the VANETs network by assisting two diverse methods of data routing. The initial one includes the delivery of data packets on the ground entirely by means of UVAR-G. The second one includes the transmission of data packets in the air depending on UVAR-S. The implementation outcomes reveal that the hybrid contact between UAVs and vehicles was perfectly appropriate for VANETs when distinguished with the traditional models[2].

In 2019, Kumari and Shylaja have established a proficient AMGRP protocol since it exploits an AHP when taking into account of numerous routing criterion like, link lifetime, mobility metric, node status and node density that were established as vital aspects for improved performance. The presented scheme was executed and the simulation outcomes have demonstrated that the modeled protocol offers better performance when evaluated with another state of the art schemes[3].
In 2018, Wang et al. have suggested a consistent routing method that intends to launch routing paths to develop the PDR without gaps in the network. Accordingly, here a routing approach depending on virtual nodes and road segments was adopted. This approach could set up the routing paths without including the network gaps, as a result, the packet loss occurred by network gaps could be evaded. Furthermore, the information could be transmitted in the shortest routing path and therefore the PDR was enhanced. At last, this method was analyzed and the data outcomes demonstrate that the PDR was efficiently enhanced[4].

In 2017, Song et al. have accomplished an SES that discretizes the probabilistic interactions into a reduced count of segments, and it further considers every segment as a sample. The period of contact among two vehicles traveling in reverse directions on the overlapping road was lesser; however, their interaction probability was superior. On the contrary, the period of the contact among two vehicles traveling in a similar direction on the overlapping road was superior; however, their interaction probability was lesser. From the analysis, the accomplished SES could attain proficient routing by in view of the above features[5].

In 2018, Daas and Salim have introduced an optimized geographic routing mechanism known as O-CLWPR, which was dependent on CLWPR and it was considered as a most capable protocol in recent times. Further, an analysis was held and the outcomes were attained, which demonstrate that the overhead load was extremely minimized and the counts of e2e delay measures and the Local Maximum Problems were also considerably minimized without weakening the PDR measure[6].

In 2017, Linda and Laurie have adopted a bandwidth evaluation approach depending on normalized throughput, that considers the PDR and interference in discrete time for each successfully delivered packet. The implemented outcomes illustrate that the approach was effectual, and could approximate the bandwidth of VANET-WSN accurately. Wide-Ranging performance analysis was held which takes into consideration the practical proliferation schemes and traffic scenarios[7].

In 2019, Lakshmana et al. have developed the conception of a smart city for enhancing city life quality. Smart cities were often introduced to gratify the safety requirements for the users and safe journeys were also ensured by adopting the smart mobility theory. Furthermore, Ant ACO was deployed for routing in VANETS over HMR distributed framework. At last, the analysis results make certain that the computational time of the adopted scheme was considered with respect to the rise in the count of a node in the HMR framework[8].

\section{B. Review}

Table 1 shows the reviews based on the optimal routing in VANETs. At first, the TROPHY algorithm was introduced which detects defective nodes and it does not need human involvement[1]. However, it needs contemplation on cost factors. 
GF technique was exploited that offered better efficiency and it also provides higher reliability, but it requires consideration of the security concerns[2].

In addition, AMGRP was deployed that offers a stable routing path and it also reduces the congestion[3]. Anyhow, there may be possibilities for frequent link interruptions. Likewise, a Reliable routing model scheme was exploited, which improves PDR and it also reduces the packet loss[4]. However, there may be feasibilities for increased routing overhead. Also, the SES scheme was employed, which offers enhanced reliability and it minimizes the delay for every cycle. However, it has to focus on the waiting time[5]. O-CLWPR classifier was exploited that provides minimal overhead load and it also offers reduced delay, anyhow, it has to consider more on geographic routing[6].

DCF was implemented which offers better PDR and it also minimizes e2e, but it has to concern more on centralized solutions[7]. At last, ACO was suggested that reduces the time for processing and it raises the count of nodes[8]. However, other metaheuristic schemes have to be considered. There, these limitations have to be considered for optimal routing in VANET systems effectively in the current research work.

TABLE I. Features and challenges of optimal routing in VANET using various techniques

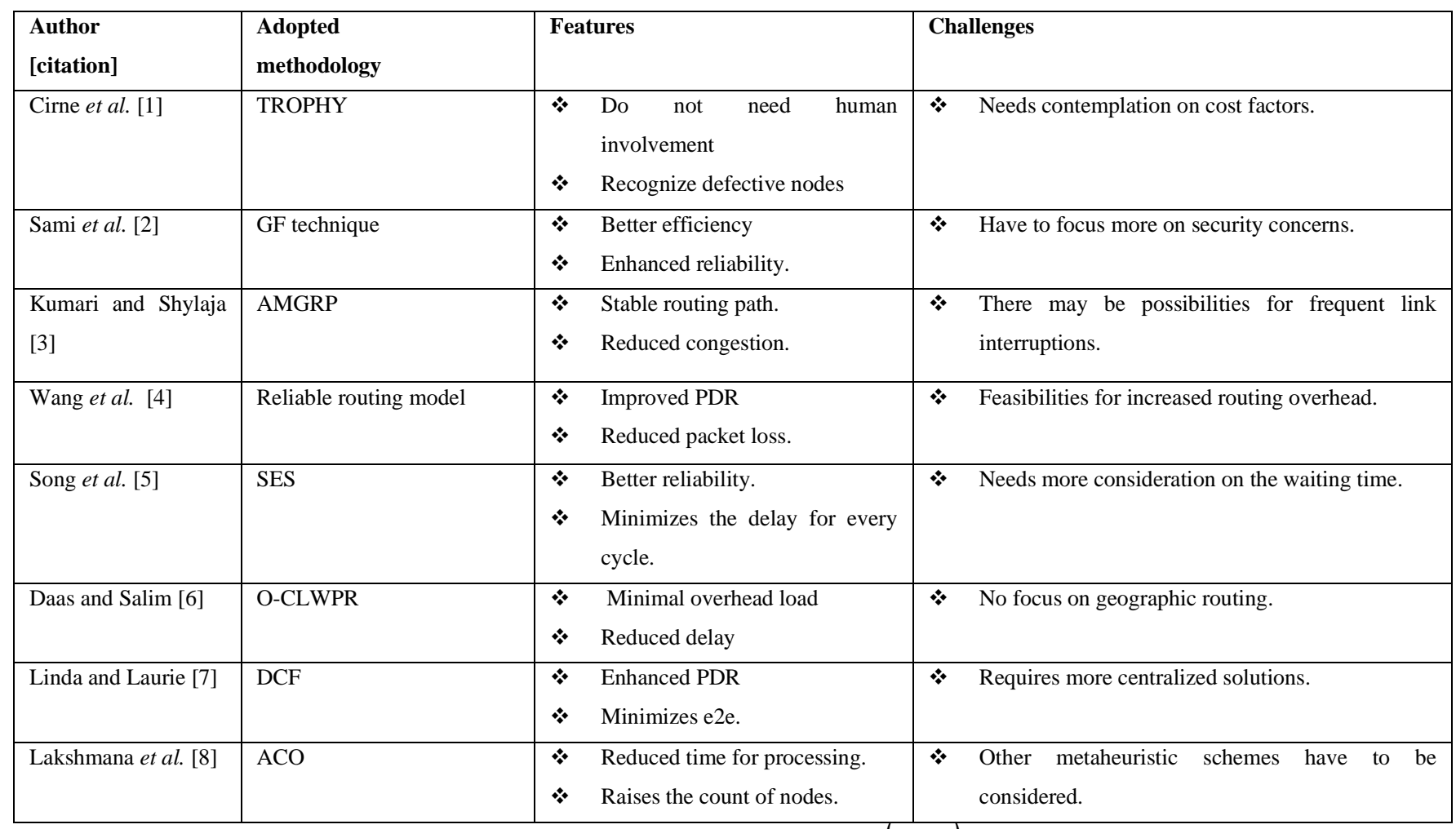

\section{OBJECTIVE MODEL FOR OPTIMAL ROUTING IN VANET}

\section{A. Objective Model}

Assume $F_{m, n}$ as a solution for the routing issue in which, $F_{m, n}: m=1,2, \ldots, N_{\text {paths }}:$ and $n=1,2, \ldots, N_{\text {nodes }}$ such that, $F_{m, n} \in\{L\}$, and $N_{\text {paths }}=N_{\text {vehicles }}$. The combination of collision cost, travel cost, QoS awareness cost and congestion cost are considered as the total routing cost, which is given in Eq. (1) [26] [27].

$$
P(F)=P_{\text {collision }}+P_{\text {travel }}+P_{\text {QoS }}+P_{\text {congestion }}
$$

Collision cost $\left(P_{\text {collision }}\right)$ : It is "the degree of striking of one vehicle violently against the other vehicle or the two or more vehicles is being assigned the same location".

Travel cost $\left(P_{\text {travel }}\right)$ : It is "the degree of time spent during the traveling which should be minimized in VANET".
QoS cost $\left(P_{Q o S}\right):$ It is "the service cost required to enable the users and applications of the network to access the new capabilities".

Congestion cost $\left(P_{\text {congestion }}\right):$ Congestion cost of VANET comprises of the stress and pollution emissions, which result from interventions between the vehicles in the traffic stream, especially when traffic volume approaches the costs of vehicle functioning and road's capacity incremental delay. In addition, better mobility could be attained by minimizing congestion. The $P_{\text {travel }}$ is determined as the cost required to travel from one locality to another locality with respect to distance or time or fuel or a group of all of them. On concerning them as a distance matrix, $P_{\text {travel }}$ could be formulated as denoted by Eq. (2). In spite of other constraints that are involved to describe the entire cost acquired by a vehicle,

Published By: 


\section{Hybridization of Monarch Butterfly and Grey Wolf Optimization for Optimal Routing in VANET}

the cost is directly proportional to the distance to be traveled by the vehicle. The remaining constraints are secondary based parameters and they are unsure. Therefore, the distance matrix was considered here for defining the entire cost. Furthermore, the other constraints are regarded with respect to congestion cost, as the total cost is based on the congestion of the particular path. In Eq. (2), $E(A, B)$ indicates the Euclidean distance among the node $A$ and $B$ portrayed from the distance matrix.

$$
P_{\text {travel }}=\sum_{m=1}^{N_{\text {path }}-1} \sum_{n=n+1}^{N_{\text {Nodes }}-1} E\left(F_{m, n-1}, F_{m, n}\right)
$$

Accordingly, $P_{\text {collision }}$ denotes the collision probability among the vehicles while they travel to their respective localities as given by Algorithm 1 .

\begin{tabular}{ll}
\hline Algorithm 1: Determination of Collision Cost \\
\hline Input & $F_{m, n} / /$ path of vehicles \\
Output & $P_{\text {collision } / / \text { collision cost }}$ \\
1 & Set $P_{\text {Collision }}=0 / /$ Initialize collision cost \\
2 & for every node till $N_{\text {Nodes }}-1 \quad \forall m$ \\
3 & Determine $V_{n} / /$ unique number of nodes available $\forall m$ \\
4 & Determine $N_{\text {coll }} / /$ Number of coding vehicles \\
5 & $P_{\text {collision }}=F_{P} \times N_{\text {coll }}+P_{\text {collision }}$ \\
6 & return $P_{\text {collision }}$
\end{tabular}

The count of the colliding vehicle $N_{\text {coll }}$ and the penalty function $F_{P}$ is multiplied under the probability of collision, here; the count of vehicles is given as $N_{\text {coll }}$, which arrive at a location at $n^{\text {th }}$ a time instant.

On the basis of the count of vehicles that are served by the $A P$ at a given instant, the congestion cost $P_{\text {congestion }}$ is found and is given in Eq. (3).

$$
\begin{aligned}
& P_{\text {congestion }}(n)=\left\{\begin{array}{l}
C_{k}^{\text {over }}(n) ; \text { if } C_{k}^{\text {over }}>0 \\
0 ; \text { otherwise }
\end{array}\right. \\
& C_{k}^{\text {over }}(n)=\sum_{\substack{m=1 \\
n \neq 1}}^{N_{\text {paths }}} C S_{k}(m, n)-C_{k}^{\lim } \\
& C S_{k}(m, n)=\left\{\begin{array}{l}
1 ; \text { if } F_{m, n} \in C_{k} \\
0 ; \text { otherwise }
\end{array}\right.
\end{aligned}
$$

Eq. (4) shows the congestion limit of $k^{\text {th }} A P$ and is indicated by $C_{k}^{\lim }$. The FIS portrays $P_{Q o S}$ by deploying the QoS constraints such as the congestion level of $A P$ and RSS.

\section{B. System Model}

The chosen network comprises of vehicles indicated as $V_{n}$, in which $n$ denotes the vehicle traveling to diverse locations $L 1, L 2, L 3, L 4, L 5, L 6, L 7, L 8$ and $L 9$. The vehicles are presumed to travel at a stable speed to arrive at the locations. An exact Access Point ( $A P$ ) encloses every location that is indicated as $A P 1, A P 2, A P 3$, and $A P 4$ with equivalent coverage area. The majority of the vehicles travel from location 4 to 6 . The issues due to coverage take place if the selection of routes is not optimal. Therefore, a correct route should be selected for attaining the utmost coverage and there should be no traffic with any collisions. For every $A P$, there is a capability to manage numerous vehicles. Going beyond a certain number of vehicles tends to cause congestion in the specific coverage area. For example, the vehicles traveling to the location $L 1, L 2, L 4$ and L5, contribute a general access point $A P 1$ and vehicles traveling to $L 3$ and $L 6$ contribute a general access point $A P 2$. The nearer $A P 3$ is shared by vehicles traveling to $L 5$ and $L 8$. It is observed that the level of congestion is high in the region $A P 4$ that encloses the $L 5, L 6, L 8$ and $L 9$. The QoS has to be improved with minimization in routing cost. The diagrammatic illustration of the vehicles traveling to diverse locations is represented in Fig. 1.

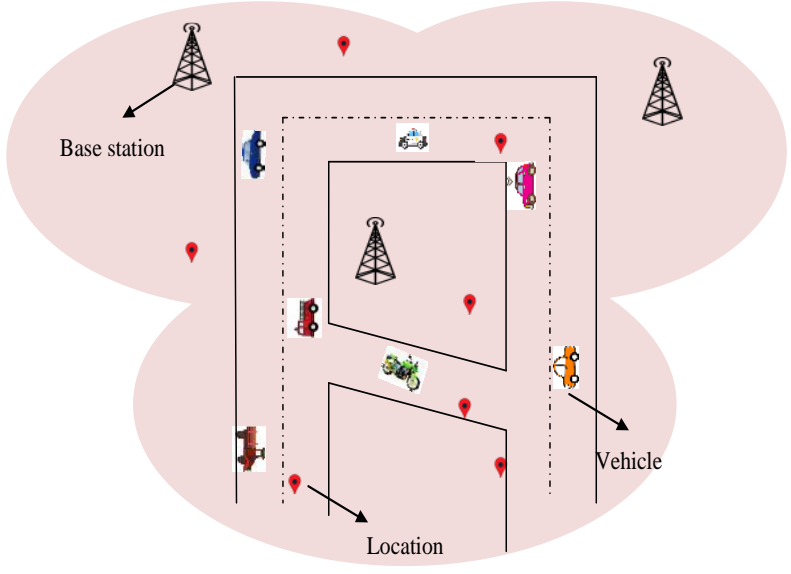

Fig. 1. Vehicles moving in various paths in a network

Consider the location, in which the chosen vehicles travel as $L_{i}$ and indicated by $L_{i}: i=1,2, \ldots, N_{\text {nodes }}$, in which, $N_{\text {nodes }}$ denotes the count of location/nodes and $i$ specifies the $i^{\text {th }}$ location/nodes. Assume $U_{j}$ as the $j^{\text {th }}$ vehicle, and it is specified by $U_{j}: j=1,2, \ldots, N_{\text {vehicles, }}$ and, $N_{\text {vehicles }}$ specifies the count of vehicles regarded in the network. The APs exploited all over the network is indicated as $A_{k}: k=1,2, \ldots, N_{A P}$, in which, $N_{A P}$ indicates the entire count of $A P$.

The network locations usually fall under the coverage area of $A P$ and the vehicles will be covered. The coverage area of every $A P$ is given by $C_{k}$, where $C_{k} \subset L$. Therefore, the cardinality of the coverage area is specified as $\left\|C_{k}\right\| \approx \frac{N_{\text {nodes }}}{N_{A P}}$.

Condition 1: $L_{i}$ lies in the coverage of $A_{k}$, only when $E\left(L_{i}, A_{k}\right)<\operatorname{rad}_{c}$ in which $\operatorname{rad}_{c}$ denotes the radius of coverage.

Theorem 1: The network's diagonal length to offer coverage to the entire nodes is specified as $=\sqrt{2 L_{\text {net }}^{2}}$, in which, $L_{\text {net }}$ denotes the network dimension.

Published By:

Blue Eyes Intelligence Engineering \& Sciences Publication

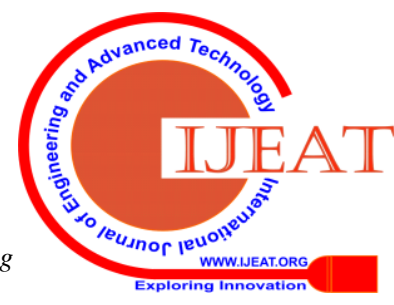


Proof: Consider that the entire nodes are located on a similar plane. Therefore, $R^{\max } \approx G^{\max }$, in which, $R^{\max }=\max \left(L_{i}(w)\right) \quad$ and $G^{\max }=\max \left(L_{i}(o)\right)$. As $L_{i}$ is indicated in the $o-w$ coordinate network, $L_{i}(o)$ indicates the $o^{\text {th }}$ coordinate of $L_{i}$ and $L_{i}(w)$ denotes the $w^{\text {th }}$ coordinate of $L_{i}$. The corner nodes are indicated by $\left(G^{\max }, 0\right)$ and $\left(R^{\max }, 0\right)$ if the network nodes are initiated from the origin. On concerning the Euclidean distance between the two points, the diagonal distance can be attained as given in Eq. (5) and Eq. (6), in which, $G^{\max }$ denotes the node's length from the origin, $L_{n e t}$.

$$
\begin{aligned}
& E^{\text {diag }}=\sqrt{\left(G^{\max }-0\right)^{2}+\left(G^{\max }-0\right)^{2}} \\
& E^{\text {diag }}=\sqrt{2 G^{\max ^{2}}}
\end{aligned}
$$

Therefore, Eq. (6) can be rewritten as Eq. (7).

$$
E^{\operatorname{diag}}=\sqrt{2 L_{\text {net }}^{2}}
$$

\section{OPTIMAL ROUTE SELECTION IN VANET USING GU-BO ALGORITHM}

\section{A. Fuzzification of QoS Factors}

Fuzzy logic deploys the non-numeric linguistic constraints for the RSS, QoS factors, QoS and congestion cost[28]. Every linguistic parameter is allocated as an arithmetical value that indicates the fuzzy membership function. If the level of congestion is high, moderate and low at fair and good RSS, the cost of QoS will be high, high and zero. However at worst RSS with a high, moderate and low level of congestion, the QoS cost is fixed as high, high and low correspondingly. When the degree of membership of congestion is fixed as low, it lies in the range of $[0,2]$, high in $[1,4]$ and moderate in $[1,2]$. On concerning the RSS parameter, the degree of membership in the range [4, $10]$ is considered as good, $[0,10]$ as fair and [0, 3] as poor. The degree of membership lying at $[0.2,1]$ is considered as high, $[0,1]$ is considered as low and $[0,0.1]$ is considered as zero in the QoS cost fuzzification. The fuzzy rules among the QoS factors and the cost concerned are given by Table II.

\section{TABLE II. Fuzzy Rules Among Qos Factors And Cost}

\begin{tabular}{|l|l|l|l|}
\hline Sl. No. & RSS & Congestion level & QoS cost \\
\hline 1 & Poor & Moderate & High \\
\hline 2 & Poor & Low & Low \\
\hline 3 & Poor & High & High \\
\hline 4 & Fair & Moderate & High \\
\hline 5 & Fair & Low & Zero \\
\hline 6 & Fair & High & High \\
\hline 7 & Good & Moderate & High \\
\hline 8 & Good & Low & Zero \\
\hline 9 & Good & High & High \\
\hline
\end{tabular}

As the main intention of the proposed routing algorithm is optimal route selection, which is attained by a new algorithm termed GU-BO. The input solution to this algorithm is the matrix, i.e. noof vehicles $\times$ noof nodes Here, nodes indicate the respective APs. The proposed algorithm is the hybridization of two conventional algorithms namely $\mathrm{MBO}$ and GWO, respectively. The description of the proposed algorithm is as follows:

\section{B. Conventional MBO}

The migration operation of the monarch butterflies are used to solve multiple optimization issues with certain characteristics such as (i) The overall population of monarch butterflies is limited to Land 1 and Land 2. (ii) The migration operator produces every single child monarch butterfly in both the regions such as Land 1 and Land 2. (iii) In the MBO approach, the parent butterfly will pass away when a new one is created so as to maintain a constant population (iv) The quality and performance of the monarch butterfly is assured even with the increased generations[24].

Migration Operator: Let $M P$ be the total number of the population, Maxgen specifies the maximum generation and $c$ indicates the ratio of butterflies in Land 1 and Land 2. For a better understanding, Land 1, and Land 2 are renamed as subpopulation1 $(S 1)$ and subpopulation $2(S 2)$.

$$
v_{i, y}^{t+1}=v_{s 2, y}^{t}
$$

Eq. (8) shows the migration process, in which, $v_{i, y}^{t+1}$ species the $y^{\text {th }}$ component $v_{i}$ of the monarch butterfly $i$ at generation $t+1$ and $v_{s_{1}, y}^{m}$ indicates the $y^{\text {th }}$ component of $v_{S_{1}}$ which is the generated location of the monarch butterfly $s_{1}$. The variable $t$ denotes the present generation. From $S 1$, Monarch butterfly $s_{1}$ is chosen arbitrarily. Using Eq. (1), the component $y$ for the new monarch butterfly is established when $s \leq c$. The value $s$ can be determined as given in Eq. (9).

$$
s=\text { ran } * \text { time }
$$

The variable time specifies migration time which is usually initiated based on 12 months of the year that can be given as 1.2. The variable ran represents an arbitrary value taken from a uniform distribution. The component $y$ for the newly produced monarch butterfly is initiated when $s>c$ as given in Eq. (10).

$$
v_{i, y}^{t+1}=v_{s 2, y}^{t}
$$

In Eq. (10), $v_{s_{2}, y}^{t}$ specifies the $y^{\text {th }}$ component of $v_{s_{2}}$ which is the newly initiated location for the monarch butterfly $s_{2}$. From $S 2$, Monarch butterfly $s_{2}$ is chosen arbitrarily. Based on the analysis done above, by regulating the ratio $c$, the stability of the direction of the migration operator can be maintained in the MBO method. The $c$ value determines if $S 1$ and $S 2$ can be chosen. The $c$ value is $5 / 12$ for the present calculation. 


\section{Hybridization of Monarch Butterfly and Grey Wolf Optimization for Optimal Routing in VANET}

Butterfly Balancing Operator: In addition to the migration operator, a balancing operator is also used to update the location of the monarch butterflies. When an arbitrarily produced value ran is less than or equivalent to $c$ for each component of the monarch butterfly $x$, the memory is then updated as shown in Eq. (11).

$$
v_{x, y}^{t+1}=v_{\text {best }, y}^{t}
$$

In Eq. (11), $v_{x, y}^{t+1}$ denotes the $y^{\text {th }}$ component of $v_{X}$ for the generation $t+1$ which denotes the location for the monarch butterfly $x$. Correspondingly, $v_{\text {best, } y}^{t}$ indicates the $y^{\text {th }}$ component of $v_{\text {best }}$ which represents the best monarch butterfly in Land 1 and Land 2. Conversely, when $c$ the value is less than ran, the memory is then updated as defined in Eq. (12).

$$
v_{x, y}^{t+1}=v_{s_{3}, y}^{t}
$$

In Eq. (12), $v_{s_{3}, y}^{t}$ specifies $y^{\text {th }}$ component of $v_{s_{3}}$ which is arbitrarily chosen in Land 2. At this point, $s_{3} \in\left\{1,2, \ldots, M P_{2}\right\}$.

$$
v_{x, y}^{t+1}=v_{x, y}^{t+1}+\lambda \times\left(f n_{y}-0.5\right)
$$

For the constraint, $r n>B A R$, the memory is then updated additionally as expressed in Eq. (13), in which, $B A R$ indicates a butterfly's balancing value. The variable $f n$ represents the monarch butterfly $x$ walk steps. It can be determined by using Levy flight as defined in Eq. (14) and (15).

$$
\begin{aligned}
& f n=\operatorname{Levy}\left(v_{x}^{t}\right) \\
& \lambda=H_{\text {max }} / t^{2}
\end{aligned}
$$

The weighting element $\lambda$ is represented based on Eq. (15), where $H_{\text {max }}$ denotes the value that at a single move, a single monarch butterfly can move the maximum walk steps. When the value of $\lambda$ is high, it represents the extended space of exploration, which maximize the impact of $f n$ on $v_{x, y}^{t+1}$ which enhance the identification of more search space, whereas the value of $\lambda$ is low, it specifies the

\begin{tabular}{|c|c|}
\hline & gorithm 2: Conventional MBO approach \\
\hline St & \\
\hline & Initialization \\
\hline & Fitness calculation \\
\hline & While $t<M P$ or if there is no optimal solution, do \\
\hline & \begin{tabular}{l|l} 
& Arrange every monarch butterflies based on its fitness
\end{tabular} \\
\hline & Split monarch butterflies into two subpopulations; \\
\hline & for $i=1$ to $M P_{1}$, do \\
\hline & \begin{tabular}{l|l} 
& Create a novel Subpopulation 1 as per migration operator.
\end{tabular} \\
\hline & end for $i$ \\
\hline & for $j=1$ to $M P_{2}$, do \\
\hline & \begin{tabular}{|l|l} 
Create a novel Subpopulation 2 as per butterfly adjusting \\
operator.
\end{tabular} \\
\hline & end for $j$ \\
\hline & Merge the two novel subpopulations into a single total population; \\
\hline
\end{tabular}
short space of exploration, which minimize the impact of fn on $v_{x, y}^{t+1}$ which enhances the exploitation process. The pseudocode of the conventional MBO model is given by algorithm 2.

\begin{tabular}{|l|l|l|}
\hline & & Compute the population based on the new updated positions; \\
\hline & & $t=t+1$ \\
\hline & end while \\
\hline & Attain the optimal solution. \\
\hline End \\
\hline
\end{tabular}

\section{Grey Wolf Optimization}

The mechanism of the GWO [25] algorithm portrays the grey wolves' hunting characteristics and their headship hierarchy. There exist 4 categorizations of grey wolves, such as $\alpha, \eta, \zeta, \omega$ that is exploited for carrying out the leadership hierarchy. Penetrating, circling, and attacking the food are the three foremost courses of action in hunting that are employed to develop optimization.

The wolves such as, $\alpha, \eta$ and $\zeta$ are considered as the foremost wolves, which are concerned in the process of hunting. Among these wolves, $\alpha$ is allocated as the leader that makes a declaration relating to the hunting process, sleeping place, time to awake, etc. On the other hand, $\eta$ and $\zeta$ holds a second and third level that assists $\alpha$ in captivating choices. Furthermore, the final level of the wolves is regarded as $\omega$ that is allocated only for eating. The encircling characteristics of the wolves are designed by means of Eq. (17) and Eq. (18), in which $X$ and $Y$ represents the coefficient vectors, $v_{p}$ refers to the position vectors of prey, $t$ denotes the current iteration and $v$ indicates the grey wolves' position vectors. The model $X$ and $Y$ is specified by Eq. (19) and Eq. (20), correspondingly, in which $a$ is a parameter which is minimized steadily from 2 to 0 for the whole iterations. The standard formulation $a$ is given by Eq. (16) and $r_{1}$ $r_{2}$ indicates the arbitrary vectors that are distributed constantly among $[0,1]$.

$$
\begin{aligned}
& a_{1}=2-1 *\left(\frac{2}{t^{\max }}\right) \\
& Z=\left|Y \cdot v_{p}^{t}-v^{t}\right| \\
& v_{p}^{(t+1)}=v_{p}^{t}-X . Z \\
& X=2 a \cdot r_{1}-a \\
& Y=2 r_{2}
\end{aligned}
$$

The arithmetical formula for portraying the hunting nature of the wolves is specified in Eq. (21) to Eq. (26), in which the final modified position of wolves is offered in Eq. (27) that offers the updated $a$ and $v$.

$$
\begin{aligned}
& Z_{\alpha}=\left|Y_{1}-v_{\alpha}-v\right| \\
& Z_{\eta}=\left|Y_{2}-v_{\eta}-v\right| \\
& Z_{\zeta}=\left|Y_{3}-v_{\zeta}-v\right| \\
& v_{1}=v_{\alpha}-X_{1} \cdot\left(Z_{\alpha}\right) \\
& v_{2}=v_{\eta}-X_{2} \cdot\left(Z_{\eta}\right) \\
& v_{3}=v_{\zeta}-X_{3} \cdot\left(Z_{\zeta}\right) \\
& v_{p}^{t+1}=\frac{v_{1}+v_{2}+v_{3}}{3}
\end{aligned}
$$




\section{Proposed model}

Despite the interesting facts about the conventional MBO approach, it includes certain disadvantages such as poorer mean fitness and worst standard values on particular benchmarks. Similarly, GWO has certain disadvantages of slow convergence, bad local searching ability and so on. Hence, to overcome the shortcomings of the conventional algorithms, this paper aims to propose a new hybrid algorithm that hybrids both MBO and GWO, respectively. The presented model is modified by incorporating the concept of GWO into the MBO algorithm. In the conventional $\mathrm{MBO}$ approach, the subpopulation 1 is generated based on the migration operator and the subpopulation 2 is generated based on the butterfly adjusting operator.

The proposed algorithm makes the modification in the generation of subpopulation 2, which is attained by the GWO update, as per Eq. (27) and thus the proposed work is termed as the GU-BO model. The pseudocode of the conventional $\mathrm{MBO}$ model is given by algorithm 3 .

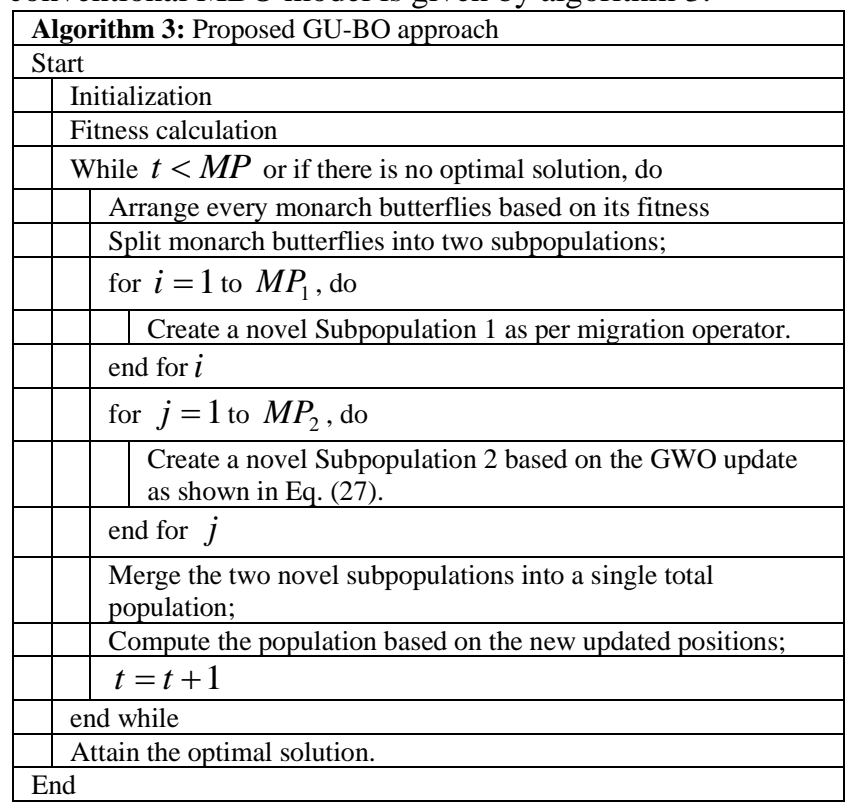

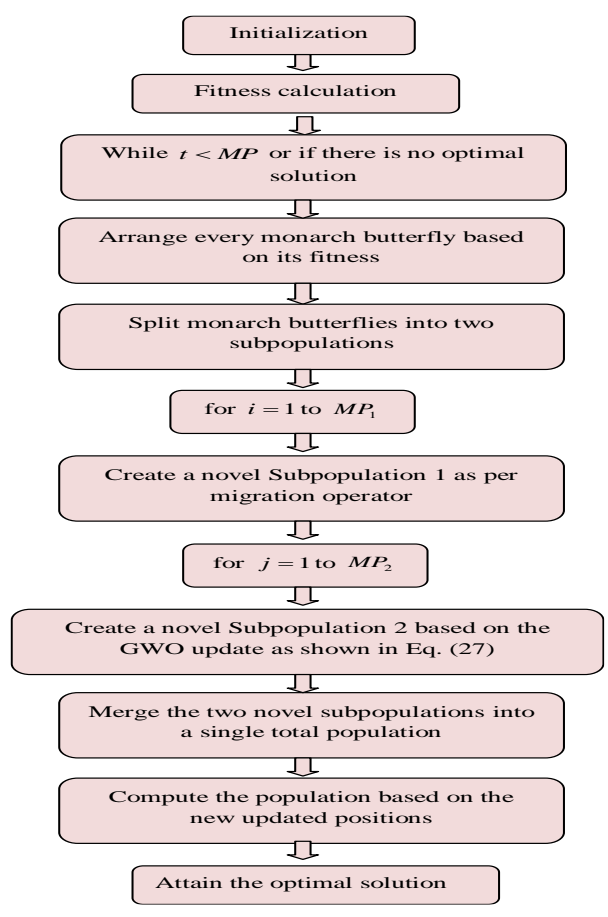

Fig. 2. Flow chart of the proposed GU-BO model

\section{RESUlt AND DiscuSSION}

\section{A. Simulation Setup}

The simulation of proposed VANET routing was done in the NS2.35 simulator. The analysis was done under four configurations by varying the instants and their respective vehicle counts namely, one access point (AP 1) with 100 vehicles, two access points (AP 2) with 200 vehicles, four access points (AP 4) with 300 vehicles, six access points (AP 6) with 400 vehicles. Here, the analysis was done by carrying out the simulation for 100 . The performance of the suggested scheme was further compared with the other conventional schemes such as PSO [29], GWO [25], FF [30], WOA [31], JA [32], MBO [24] and MC-JA [33] in terms of the cost function. The simulation results for the four above mentioned configurations were given by Fig. 3.

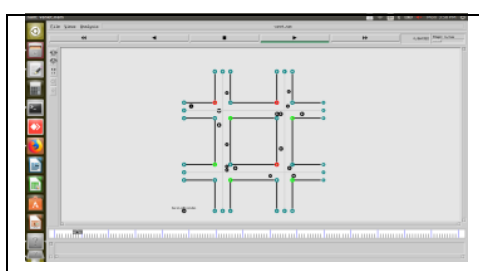

(a)

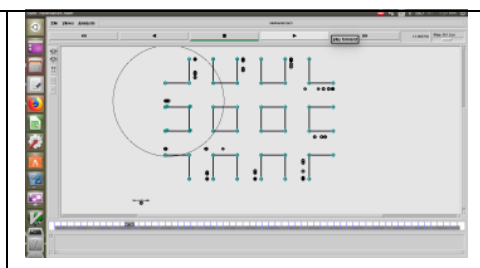

(b)

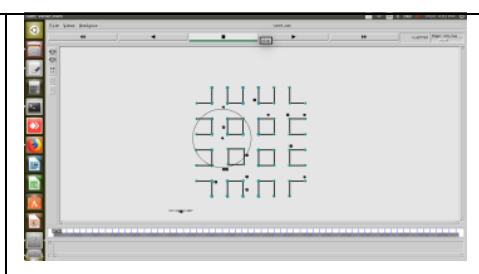

(c)

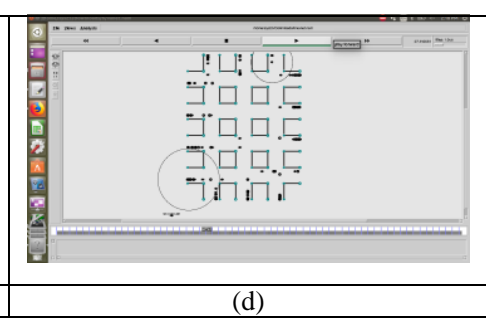

(d)

Fig. 3. Simualtion result for (a) configuration 1 (one access point (AP 1) with 100 vehicles) (b) configuration 2 (two access points (AP 2) with 200 vehicles) (c) configuration 3 (four access points (AP 4) with 300 vehicles) (d) configuration 4 (six access points (AP 6) with 400 vehicles)

\section{B. Analysis of Travel Cost}

The analysis on travel cost for the proposed and conventional models for the four configurations namely, AP 1 with 100 vehicles, AP 2 with 200 vehicles, AP 4 with 300 vehicles, AP 6 with 400 vehicles is given by Fig. 4. From the analysis, reduced cost values are attained by the presented model when compared to other schemes. From
Fig. 4(c), configuration 3 of the proposed model is $6.66 \%$, $5.1 \%, 9.67 \%$, 5.9\%, $1.28 \%, 4.43 \%$, and $0.52 \%$ better than PSO, FF, GWO, WOA, JA, MBO, and MC-JA approaches at $20^{\text {th }}$ iteration.

Published By:

Blue Eyes Intelligence Engineering \& Sciences Publication 
Likewise, from Fig. 4(d), configuration 3 of the presented GU-BO scheme at $100^{\text {th }}$ iteration has attained less travel cost, which is $43.84 \%, 28.43 \%, 44.78 \%, 28.85 \%$, $10.65 \%, 21.92 \%$ and $6.11 \%$ superior to PSO, FF, GWO,
WOA, JA, MBO, and MC-JA models. Similarly, all other configurations have proved the superiority of proposed work over the traditional schemes with respect to reduced travel costs.

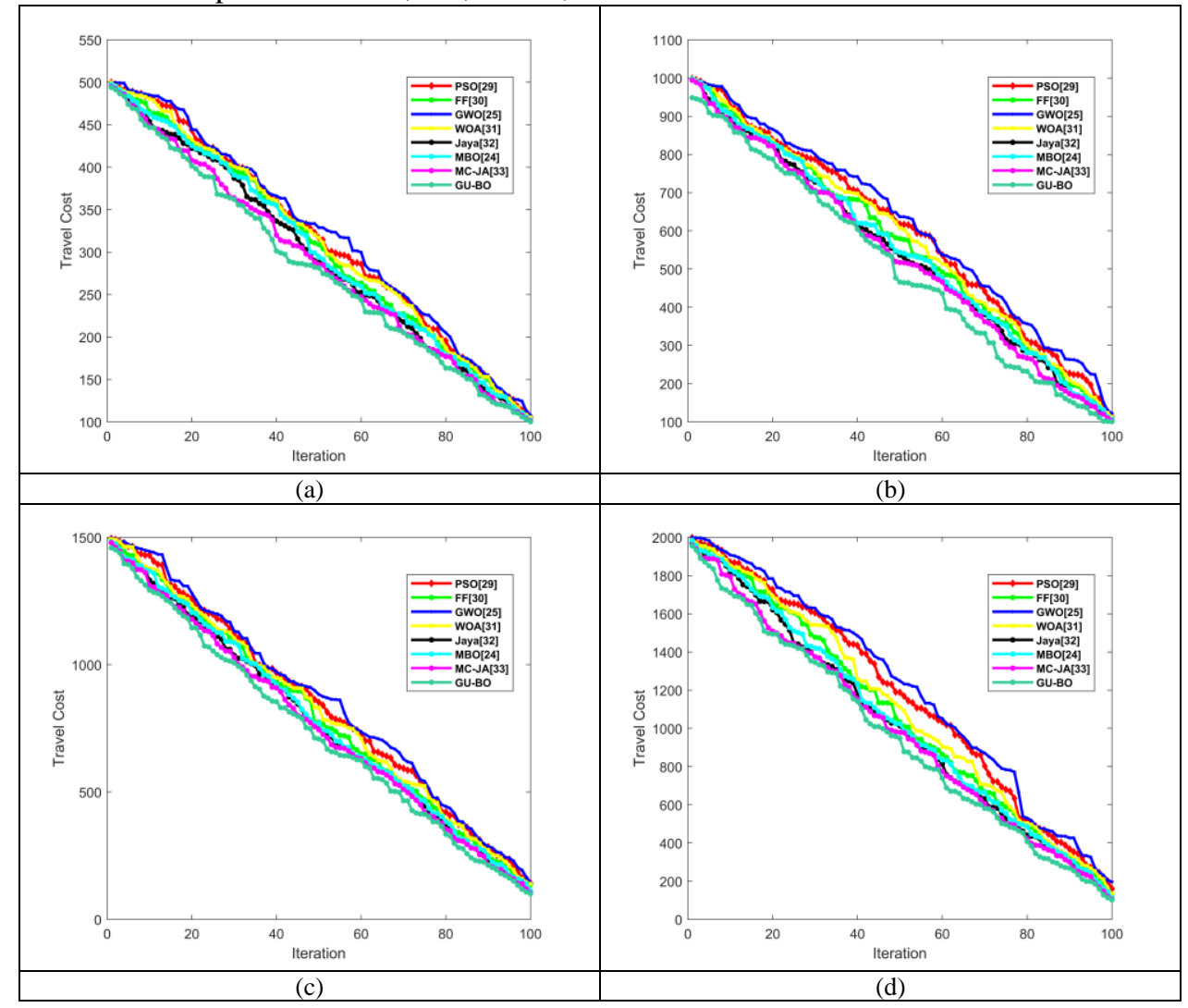

Fig. 4. Analysis on travel Cost under four configurations (a) one access point (AP 1) with 100 vehicles (b) two access points (AP 2) with 200 vehicles (c) four access points (AP 4) with 300 vehicles (d) six access points (AP 6) with 400 vehicles

\section{Analysis of Collision Cost}

The collision cost analysis for the proposed GU-BO scheme when evaluated over the conventional models for the four configurations such as AP 1 with 100 vehicles, AP 2 with 200 vehicles, AP 4 with 300 vehicles, AP 6 with 400 vehicles is illustrated in Fig. 5. From the experimental analysis, minimal collision cost values are found to be attained by the presented model over other existing schemes. From Fig. 5(c), in configuration 1, the proposed model is $4.98 \%$, $3.08 \%, 3.13 \%, 4.89 \%, 2.27 \%, 2.66 \%$, and $1.13 \%$ better than PSO, FF, GWO, WOA, JA, MBO, and MC-JA approaches at 20th iteration. Moreover, from Fig. 5(b), configuration 2 of the developed GU-BO scheme at 40th iteration proves the cost minimization of proposed model, which is $11.7 \%, 6.99 \%, 8.83 \%, 11.19 \%, 2.69 \%$, $3.07 \%$ and $0.7 \%$ superior to PSO, FF, GWO, WOA, JA, $\mathrm{MBO}$, and MC-JA models. Thus the betterment of the presented scheme is offering a reduced minimized collision cost function is proved in an effective manner. 


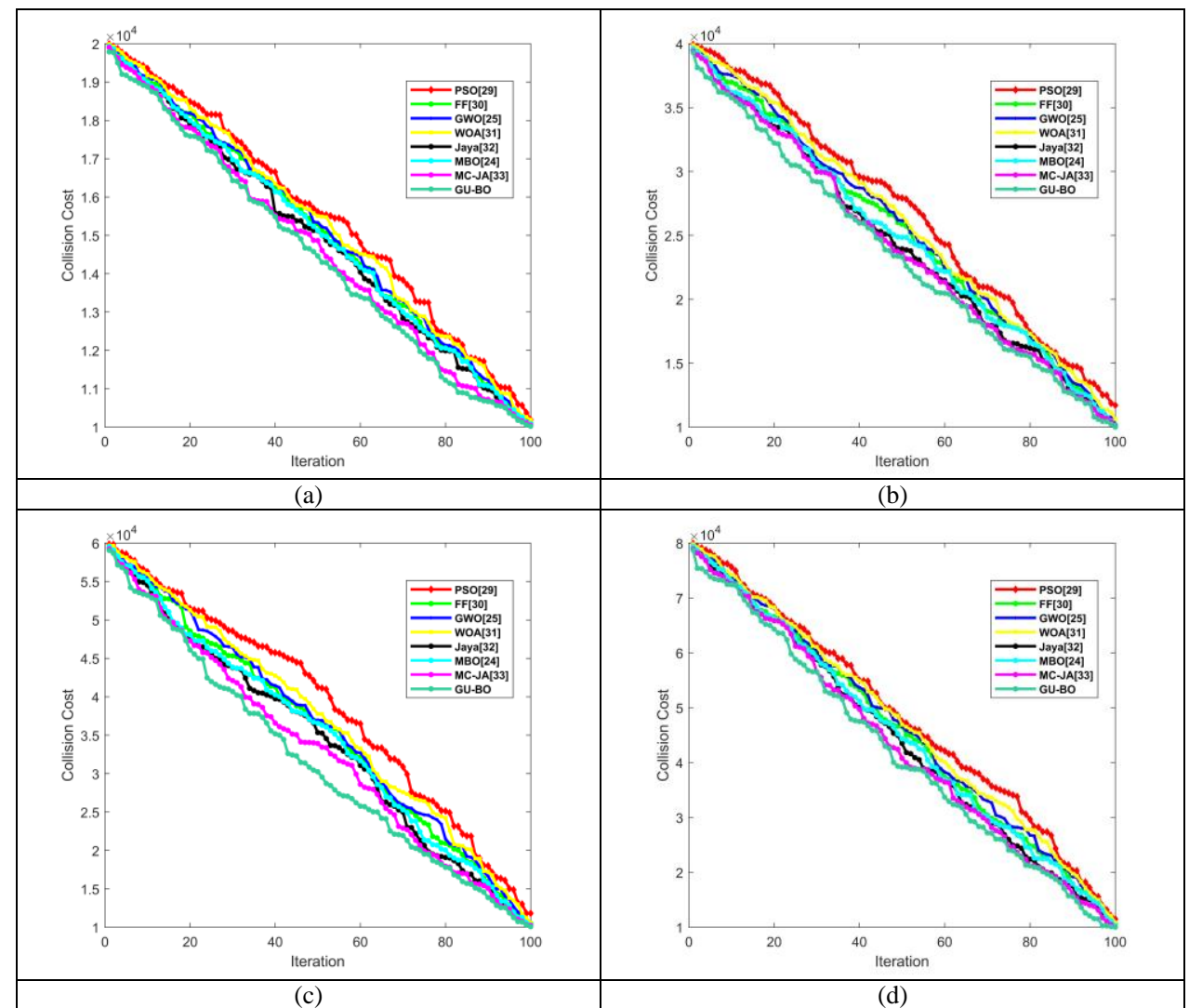

Fig. 5. Analysis on collision Cost under four configurations (a) one access point (AP 1) with 100 vehicles (b) two access points (AP 2 ) with 200 vehicles (c) four access points (AP 4) with 300 vehicles (d) six access points (AP 6) with 400 vehicles

\section{Analysis of Congestion Cost}

Fig. 6 shows the congestion cost analysis under all four configurations of both the proposed GU-BO model and the conventional methods. The minimal congestion cost is attained by the adopted model which is proved from the simulation outcomes. From Fig. 6(a), the congestion cost at 20th iteration for configuration 1 for the proposed GU-BO model is $1.38 \%, 3.47 \%, 6.55 \%, 5.79 \%, 4.54 \%, 2.19 \%$ and
$1 \%$ superior to PSO, FF, GWO, WOA, JA, MBO, and MCJA models. Also, the presented scheme at 60th iteration for configuration 1 is $2.63 \%$, 4.89\%, 5.92\%, 5.31\%, 5.19\%, $3.62 \%$ and $2.54 \%$ better than PSO, FF, and GWO, WOA, JA, MBO, and MC-JA models. Thus, the enhanced outcome of the presented model has been confirmed in terms of the congestion cost.

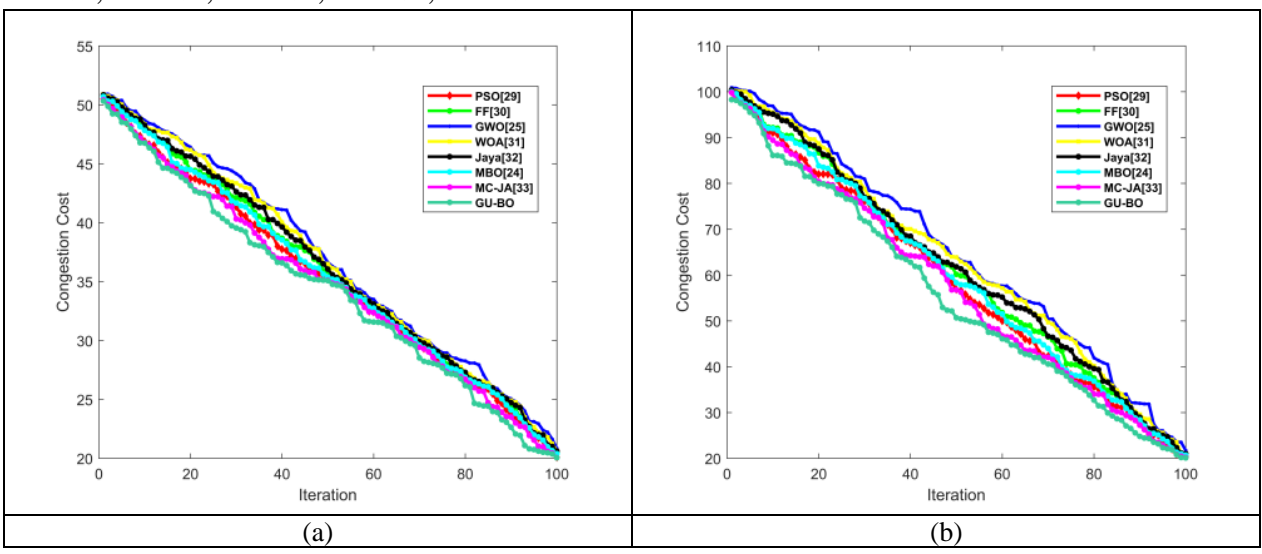




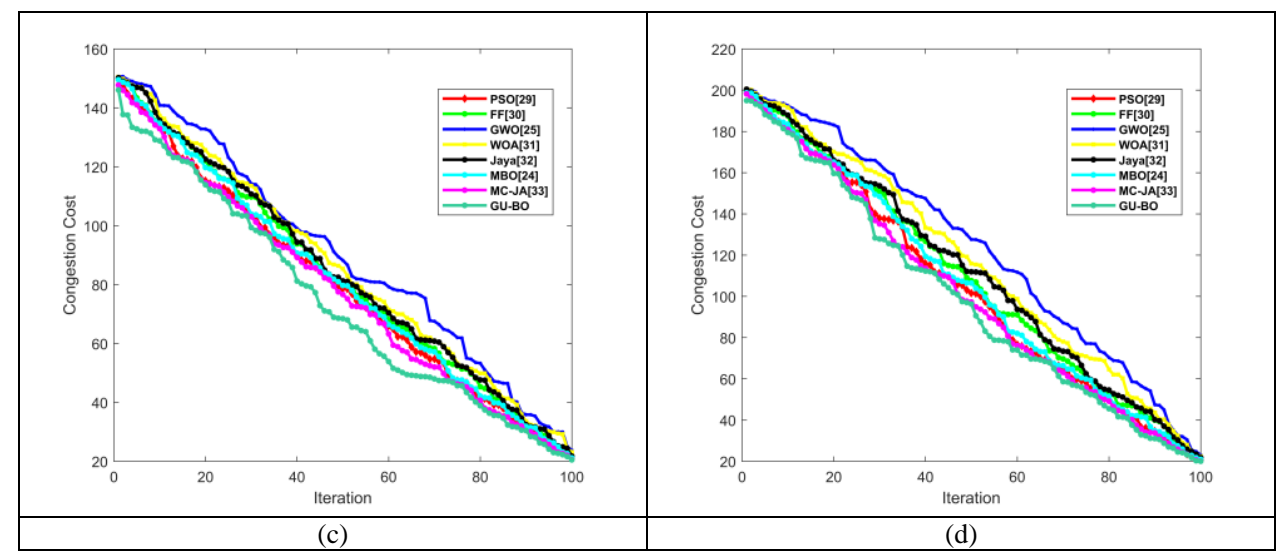

Fig. 6. Analysis on Congestion Cost under four configurations (a) one access point (AP 1) with 100 vehicles (b) two access points (AP 2 ) with 200 vehicles (c) four access points (AP 4) with 300 vehicles (d) six access points (AP 6) with 400 vehicles

\section{E. Analysis of QoS Awareness Cost}

The analysis of QoS awareness cost for both the proposed GU-BO model and the traditional methods for the four configurations are shown in Fig. 7. From the experimental outcomes, reduced QoS cost is achieved by the presented scheme. From Fig. 7(b), the QoS awareness cost at 40th iteration for configuration 2 for the proposed GU-BO model is $18.52 \%, 23.21 \%, 7.88 \%, 7.5 \%, 15.04 \%$,
7.5\% and $6.99 \%$ superior to PSO, FF, GWO, WOA, JA, MBO, and MC-JA models. Also, from Fig. 7(d), the presented scheme at 20th iteration for configuration 4 is $8.25 \%, 8.53 \%, 5.19 \%, 1.31 \%, 6.09 \%, 2.18 \%$, and $1.62 \%$ better than PSO, FF, and GWO, WOA, JA, MBO, and MCJA models. Therefore, the superiority of the introduced model in terms of QoS cost is verified.

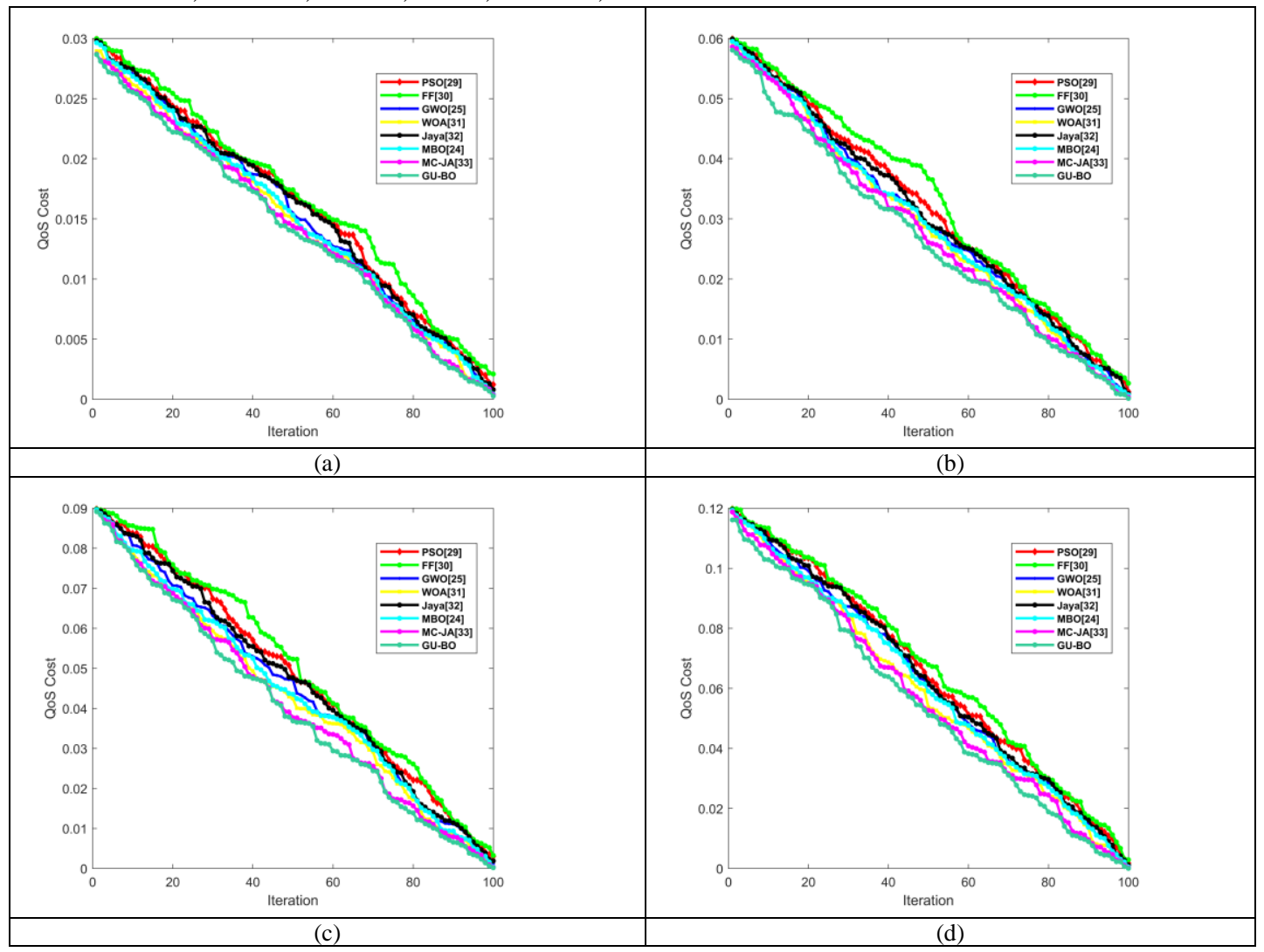

Fig. 7. Analysis on QoS awareness Cost under four configurations (a) one access point (AP 1) with 100 vehicles (b) two access points (AP 2) with 200 vehicles (c) four access points (AP 4) with 300 vehicles (d) six access points (AP 6) with 400 vehicles

\section{F. Analysis of Convergence Cost}

Fig. 8 demonstrates the convergence analysis of the implemented GU-BO model for four configurations like AP 1 with 100 vehicles, AP 2 with 200 vehicles, AP 4 with 300 vehicles, AP 6 with 400 vehicles. From the experimentation, better outcomes are accomplished by the proposed GU-BO model when compared to the prevailing schemes. From Fig. 8(a), the suggested scheme at 20th iteration for configuration 1 is $5.14 \%, 5.11 \%, 7.5 \%, 6.46 \%$, $3.99 \%, 4.77 \%$ and $2.3 \%$ superior to PSO, FF, GWO, WOA, JA, MBO, and MC-JA approaches with least cost function.

Published By:
Blue Eyes Intelligence Engineering \& Sciences Publication 
In addition, from Fig.8(d), the proposed GU-BO algorithm is $6.66 \%, 5.1 \%, 9.67 \%, 5.9 \%, 1.28 \%, 4.43 \%$, and $0.52 \%$ better than PSO, FF, GWO, WOA, JA, MBO, and MC-JA approaches at 60th iteration for configuration 4. Therefore, it is known from the experimental results that the proposed
GU-BO has the capability to offer better routing with minimal cost.

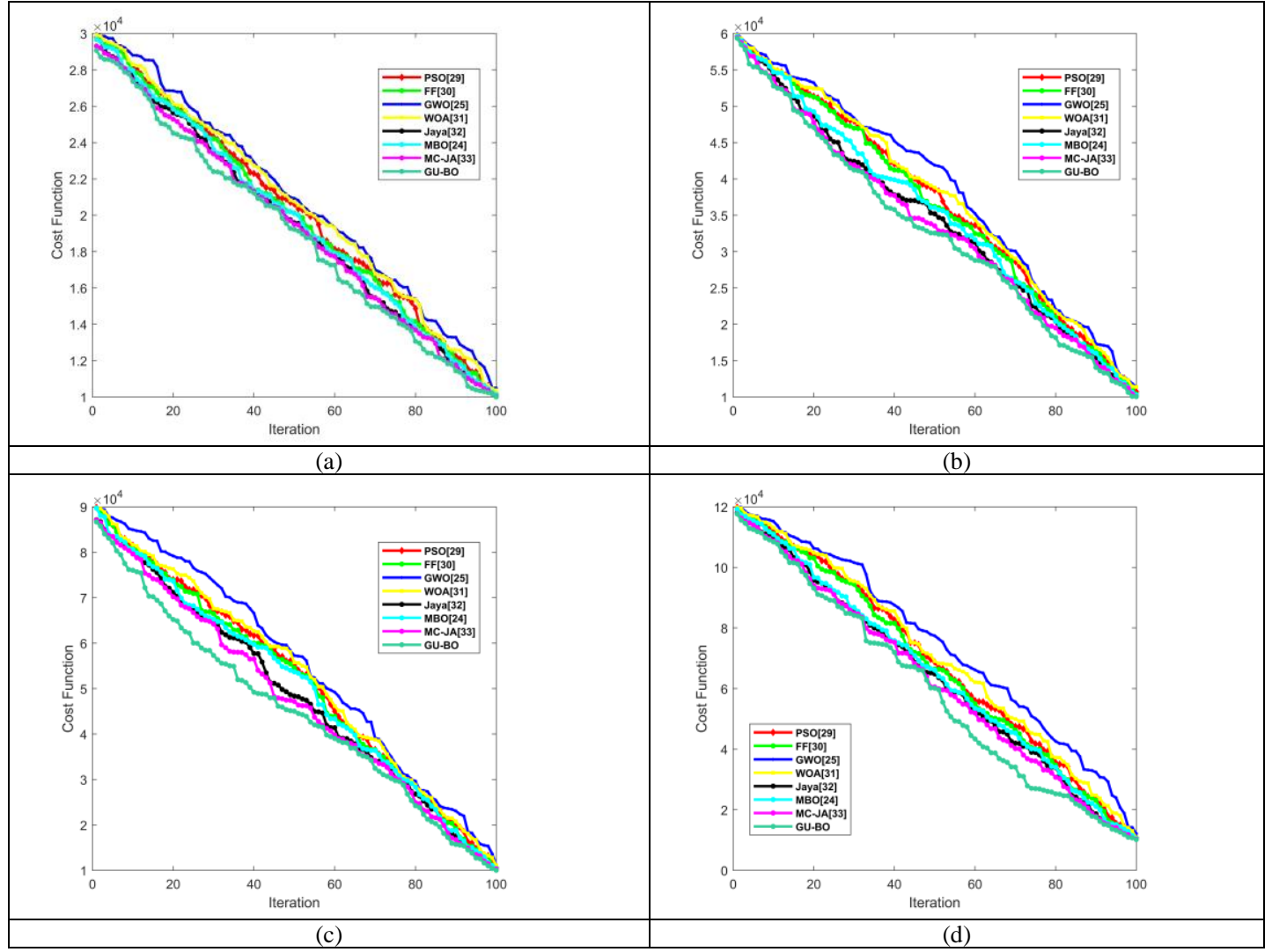

Fig. 8. Analysis on convergence Cost under four configurations (a) one access point (AP 1) with 100 vehicles (b) two access points (AP 2 ) with 200 vehicles (c) four access points (AP 4) with 300 vehicles (d) six access points (AP 6) with 400 vehicle

\section{CONCLUSION}

This paper has presented an enhanced VANET routing by a new GU-BO model concerning the network parameters namely, congestion cost, travel cost, collision cost, and QoS awareness cost. A cost model was modeled that tackles vehicle routing problems by considering the Fuzzification of the QoS factor along with the aforementioned cost parameters. The main aim of the proposed model is to discover an optimal route with a reduced routing cost model. For determining the optimal route, a hybrid algorithm known as GU-BO was introduced. From the analysis, better outcomes are accomplished by the proposed GU-BO model when compared to the prevailing schemes. From the analysis, the suggested scheme at 20th iteration for configuration 1 was $5.14 \%$, 5.11\%, 7.5\%, 6.46\%, $3.99 \%, 4.77 \%$ and $2.3 \%$ superior to PSO, FF, GWO, WOA, JA, MBO, and MC-JA approaches. In addition, the proposed GU-BO algorithm was 6.66\%, 5.1\%, 9.67\%, $5.9 \%, 1.28 \%, 4.43 \%$, and $0.52 \%$ better than PSO, FF, GWO, WOA, JA, MBO, and MC-JA approaches at 60th iteration for configuration 4 . Thus, the improvement of the proposed GU-BO approach in determining the optimal route was proved over other models.

\section{REFERENCES}

1. Pedro Cirne, André Zúquete, Susana Sargento, "TROPHY: Trustworthy VANET routing with group authentication keys", Ad Hoc Networks, vol. 71, pp. 45-67, 15 March 2018.

2. Omar Sami Oubbati, Abderrahmane Lakas, Fen Zhou, Mesut Güneş, Mohamed Bachir Yagoubi, "Intelligent UAV-assisted routing protocol for urban VANETs”, Computer Communications, vol. 107, pp. 93-111, 15 July 2017.

3. N. V. Dharani Kumari, B. S. Shylaja, "AMGRP: AHP-based Multimetric Geographical Routing Protocol for Urban environment of VANETs", Journal of King Saud University - Computer and Information Sciences, vol. 31, no. 1, pp. 72-81, January 2019.

4. Xiaonan Wang, Dong Wang, Qi Sun, "Reliable routing in IP-based VANET with network gaps", Computer Standards \& Interfaces, vol. 55, pp. 80-94, January 2018.

5. Chao Song, Jie Wu, Ming Liu, Huanyang Zheng, "Efficient routing through discretization of overlapped road segments in VANETs", Journal of Parallel and Distributed Computing, vol. 102, pp. 57-70, April 2017.

6. Mohamed Skander Daas, Salim Chikhi, "Optimizing geographic routing protocols for urban VANETs using stigmergy, social behavior and adaptive C-n-F mechanisms: An optimized CLWPR", Vehicular Communications, vol. 14, pp. 97-108, October 2018.

7. Linda F. Mohaisen, Laurie L. Joiner, "Interference aware bandwidth estimation for load balancing in EMHR-energy based with mobility concerns hybrid routing protocol for VANET-WSN communication", Ad Hoc Networks, vol. 66, pp. 1-15, November 2017. 


\section{Hybridization of Monarch Butterfly and Grey Wolf Optimization for Optimal Routing in VANET}

8. S. K. Lakshmanaprabu, K. Shankar, Rani S. Sheeba, Abdulhay Enas, J. Uthayakumar, "An effect of big data technology with ant colony optimization based routing in vehicular ad hoc networks: Towards smart cities", Journal of Cleaner Production, 12 January 2019.

9. C. Wu, S. Ohzahata and T. Kato, "Flexible, Portable, and Practicable Solution for Routing in VANETs: A Fuzzy Constraint QLearning Approach," IEEE Transactions on Vehicular Technology, vol. 62, no. 9, pp. 4251-4263, Nov. 2013.

10. J. Nzouonta, N. Rajgure, G. Wang and C. Borcea, "VANET Routing on City Roads Using Real-Time Vehicular Traffic Information," IEEE Transactions on Vehicular Technology, vol. 58, no. 7, pp. 3609-3626, Sept. 2009.

11. J. Huang, "Accurate Probability Distribution of Rehealing Delay in Sparse VANETs," IEEE Communications Letters, vol. 19, no. 7, pp. 1193-1196, July 2015.

12. R. Shahidi and M. H. Ahmed, "Probability Distribution of End-toEnd Delay in a Highway VANET," IEEE Communications Letters, vol. 18, no. 3, pp. 443-446, March 2014.

13. C. - Huang, H. - Ku and H. - Kung, "Efficient power-consumptionbased load-sharing topology control protocol for harsh environments in wireless sensor networks," IET Communications, vol. 3, no. 5, pp. 859-870, May 2009.

14. D. Ludovico Guidoni, F. Sumika Hojo Souza, J. Ueyama and L. Aparecido Villas, "RouT: A Routing Protocol based on Topologies for Heterogeneous Wireless Sensor Networks," IEEE Latin America Transactions, vol. 12, no. 4, pp. 812-817, June 2014.

15. YanSHI, Xiao-yeJIN, Shan-zhiCHEN," AGP: an anchor-geography based routing protocol with mobility prediction for VANET in city scenarios", The Journal of China Universities of Posts and Telecommunications, vol.18, no.1, September 2011, pp.112-117.

16. YuDING, Ya-Zhi Liu, Xiang-yang gong, Wen-dongWANG," Road traffic and geography topology-based opportunistic routing for VANETs", The Journal of China Universities of Posts and Telecommunications, vol.21, no.4, August 2014, pp.32-39.

17. Akshat Gaurav, Awadhesh Kumar Singh," Lightweight approach for secure backbone construction for MANETs", Journal of King Saud University - Computer and Information Sciences, Available online 6 June 2018.

18. Antesar M.Shabut, M. ShamimKaiser, Keshav P.Dahal, WenbingChen, "A multidimensional trust evaluation model for MANETs", Journal of Network and Computer Applications, Available online 20 July 2018.

19. G.G.Md.Nawaz Ali, Peter Han Joo Chong, Syeda Khairunnesa Samantha, EdwardChan," Efficient data dissemination in cooperative multi-RSU Vehicular Ad Hoc Networks (VANETs)", Journal of Systems and Software, vol.117, July 2016, pp.508-527.

20. Saaidal Razalli Azzuhri, Harith Ahmad, Marius Portmann, Ismail Ahmed, Ranjana Pathak," An Efficient Hybrid MANET-DTN Routing Scheme for OLSR", Wireless Personal Communications, August 2016, vol.89, no.4, pp 1335-1354.

21. Tasneem Darwish, Kamalrulnizam Abu Bakar, "Traffic aware routing in vehicular ad hoc networks: characteristics and challenges", Telecommunication Systems, March 2016, vol.61, no.3, pp 489-513.

22. Elham Moridi, Hamid Barati," RMRPTS: a reliable multi-level routing protocol with tabu search in VANET", Telecommunication Systems, May 2017, vol.65, no.1, pp 127-137.

23. Y. Sun, Q. Jiang and M. Singhal, "A Hill-Area-Restricted Geographic Routing Protocol for Mobile Ad Hoc and Sensor Networks," The Computer Journal, vol. 55, no. 8, pp. 932-949, Aug. 2012.

24. Gai-Ge Wang, Suash Deb, and Zhihua Cui, "Monarch butterfly optimization", Neural Computing and Applications, pp 1-20, February 2015

25. Seyedali Mirjalili a, Seyed Mohammad Mirjalili, Andrew Lewis, "Grey Wolf Optimizer", Advances in Engineering Software, vol. 69, pp.46-61, 2014.

26. S. Zeadally, R. Hunt, Y.-S. Chen, A. Irwin, and A. Hassan, "Vehicular ad hoc networks (VANETs): status, results, and challenges," Telecommun. Syst. vol. 50, no 4, pp. 217-241, 2012.

27. C. Wu, S. Ohzahata, and T. Kato, "Flexible, Portable, and Practicable Solution for Routing in VANETs: A Fuzzy Constraint QLearning Approach," IEEE Transactions on Vehicular Technology, vol. 62, no. 9, pp. 4251-4263, November 2013.

28. G.J. Klir, U.St. Clair, and Y. Bo, "Fuzzy Set Theory: Foundations and Applications," Englewood Cliffs, NJ, USA: Prentice-Hall, 1997.

29. Junhao Zhang, Pinqi Xia, "An improved PSO algorithm for parameter identification of nonlinear dynamic hysteretic models", Journal of Sound and Vibration, vol. 389, pp. 153-167, 17 February 2017.
30. Iztok Fister, Iztok Fister, Xin-She Yang, Janez Brest, "A comprehensive review of firefly algorithms", Swarm and Evolutionary Computation, vol. 13, pp. 34-46, December 2013

31. Seyedali Mirjalili, Andrew Lewis, "The Whale Optimization Algorithm", Advances in Engineering Software, vol. 95, pp. 51-67, May 2016.

32. H. M. Pandey, "Jaya a novel optimization algorithm: What, how and why?," 2016 6th International Conference - Cloud System and Big Data Engineering (Confluence), Noida, pp. 728-730, 2016.

33. Tony Santhosh, "Optimal Routing in VANET using Improved Metaheuristic Approach: A Variant of JAYA", to be published.

\section{AUTHORS PROFILE}

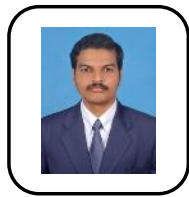

G.Tony Santhosh is an Assistant Professor of ECE department, Alpha College of Engineering, Chennai.His area of interest is Vehicular networks. He has published 2 international conference papers and 2 international journal papers.

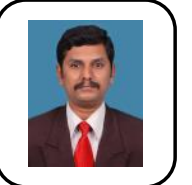

Dr.S.Dhandapani is a Professor of ECE department, Saveetha Engineering College, Chennai. His arear of interest is Digital Image Processing. He is guiding more than 5 research scholars with a publication profile of 12 international conference papers and 20 international journal papers. 